\title{
GERENCIAMENTO DE RESÍDUOS QUÍMICOS E ÁGUAS SERVIDAS NO LABORATÓRIO DE ISÓTOPOS ESTÁVEIS DO CENA/USP
}

\author{
José Albertino Bendassolli*, Everaldo Máximo, Glauco Arnold Tavares e Raquel de Fátima Ignoto \\ Centro de Energia Nuclear na Agricultura, Universidade de São Paulo, CP 96, 13400-970 Piracicaba - SP
}

Recebido em 28/6/02; aceito em 5/11/02

\begin{abstract}
CHEMISTRY WASTE AND RESIDUES WATERS MANAGEMENT AT THE STABLE ISOTOPE LABORATORY OF THE CENA/USP. A system for disposal and recovery of the main effluents and chemical waist from isotope separation plants and enriched compounds- ${ }^{15} \mathrm{~N}$ and ${ }^{34} \mathrm{~S}$ production has been carried out at the Stable Isotope Laboratory (LIE) of the CENA/USP. Around four hundred thousand liters of effluents has been recovered yearly. Among the recovered chemical wastes, the more relevant are: ammonia; brome; ammonium and sodium sulfate; sodium hydroxide; sulfur dioxide; and hydrochloric acid. Chemical wastes containg recoverable heavy metals $(\mathrm{Ag}, \mathrm{Cr}$ and $\mathrm{Cu}$ ) and solvents (methanol, ethanol and acetone) are processed and recovered. Gaseous emissions, mainly $\mathrm{H}_{2} \mathrm{~S}$ are used for recovery of heavy metals solutions. The minimization of the residues waters, as well the reduction of electric energy consume was established using a water deionization system. A cost/effect balance of the process is reported.
\end{abstract}

Keywords: residues; treatment; management.

\section{INTRODUÇÃO}

De um modo geral, desprezando-se os graus de periculosidade, a atividade humana sempre gerou alguma forma de resíduo, alguns deles nocivos ao meio ambiente e, por conseguinte, ao próprio homem. Entretanto, alguns fatores, dentre os quais podem-se destacar aqueles relacionados ao crescimento populacional e desenvolvimento industrial, acentuaram notavelmente a geração de resíduos no mundo.

Embora, na natureza, a exposição constante a produtos químicos seja comum uma vez que plantas, animais e mesmo rochas e solos emitem gases e substâncias orgânicas para o meio ambiente, a atividade antropogênica aumentou consideravelmente a liberação destes materiais. Isso fez com que organismos vivos que, em outros tempos, estavam em equilíbrio com as exudações naturais, estejam no presente expostos a um estresse químico ${ }^{1}$.

Nas últimas décadas, tornou-se evidente a necessidade de se tomarem providências para o controle da emissão de resíduos, evitando que os recursos naturais como água, solo e ar tornem-se ainda mais degradados. Os efeitos desta geração indiscriminada, que atingem também o homem, têm levado a sociedade a uma maior conscientização do real perigo para a sua subsistência. Eventos como o ocorrido em 1956 na cidade costeira japonesa de Minamata, onde o efluente industrial contendo mercúrio provocou acentuada mortandade de peixes nessa baía, os quais acabaram sendo ingeridos pela população local, ocasionando um total de 887 mortes e 2209 casos registrados de doenças relacionadas ao sistema nervoso cen$\operatorname{tral}^{2}$, dentre outros, têm servido de alerta para que a preocupação com as questões ambientais passe do discurso para a prática, nos dias atuais.

Sabe-se que as indústrias, principalmente aquelas que utilizam produtos químicos em seus processos produtivos, são as maiores responsáveis pela geração de resíduos perigosos e o grande alvo de

*e-mail: jab@cena.usp.br cobrança e fiscalização pela sociedade e pelos órgãos competentes.

$\mathrm{Na}$ sua totalidade, os centros de formação de recursos humanos (universidades e escolas) geram cerca de $1 \%$ dos resíduos perigosos em um país desenvolvido como os Estados Unidos ${ }^{3}$. Ao contrário das unidades industriais, estes resíduos caracterizam-se por apresentarem volume baixo e elevada diversidade, o que dificulta a padronização das formas de tratamento e disposição. Nos últimos anos a preocupação com os resíduos gerados nas indústrias químicas, instituições de pesquisa e ensino e órgãos governamentais tem aumentado consideravelmente e, sempre que possível, existe um empenho para a melhor disposição e recuperação dos resíduos gerados em vários processos, com o objetivo de torná-los úteis novamente ${ }^{4-24}$.

Ainda que este volume seja reduzido, as universidades não podem nem devem ignorar sua posição de geradora de resíduos ${ }^{3,25-27}$. Um dos motivos dessa necessidade é que as universidades exercem papel fundamental quando avaliam os impactos ambientais provocados por outras unidades geradoras de resíduos fora de seus limites físicos. Desta forma, o não tratamento de seus próprios rejeitos, mitigaria a credibilidade das universidades perante a sociedade e os órgãos públicos competentes ${ }^{27}$. Outro motivo, e talvez o principal, é que o maior benefício proporcionado por um programa de gerenciamento de resíduos nestas unidades está relacionado ao treinamento dos estudantes, formação profissional de técnicos de laboratórios e professores, capacitando-os a trabalharem dentro de normas apropriadas de gerenciamento de produtos químicos ${ }^{3}$.

O Laboratório de Isótopos Estáveis (LIE-CENA/USP) é responsável por toda a produção nacional de compostos enriquecidos nos isótopos de ${ }^{15} \mathrm{~N} \mathrm{e}{ }^{34} \mathrm{~S}$. Com o aumento significativo do consumo desses compostos, a produção foi ampliada em até vinte vezes, nos últimos cinco anos, gerando um elevado volume de efluentes químicos, sendo necessário instituir um programa de gerenciamento dos mesmos. Dentro deste contexto, o laboratório desenvolve um programa de gerenciamento dos resíduos proporcionando condições de desenvolvimento de tecnologia de separação e produção de inúmeros compostos enriquecidos nos isótopos ${ }^{15} \mathrm{Ne} \mathrm{e}^{34} \mathrm{~S}$ (tecnologia não repassada por países que as detém). Cabe destacar que o gerenciamento destes resíduos químicos agrega valor ambiental e, conseqüentemente, eco- 
nômico, caracterizando-o como tecnologia limpa, em conformidade com os conceitos de "química limpa" e os padrões internacionais exigidos pela legislação ambiental.

\section{Produção de compostos enriquecidos nos isótopos de ${ }^{15} \mathrm{~N}$ e/ou ${ }^{34}$ S no LIE}

O LIE-CENA/USP domina metodologia para obtenção e síntese de quinze compostos nitrogenados enriquecidos em ${ }^{15} \mathrm{~N}$ e nove compostos marcados no isótopo ${ }^{34} \mathrm{~S}$. Os compostos enriquecidos são obtidos principalmente a partir da ${ }^{15} \mathrm{NH}_{3 \mathrm{aq}}$ e do ${ }^{34} \mathrm{SO}_{2 \mathrm{q}}$. Estes dois compostos são produzidos nas linhas de separação isotópica, constituídas por colunas de cromatografia de troca iônica. A linha de separação dos isótopos de ${ }^{15} \mathrm{~N}$ consta de três sistemas de colunas de resina catiônica Dowex 50W X8 em cascata para produção de ${ }^{15} \mathrm{NH}_{4}^{+}$ com elevado enriquecimento ${ }^{28}$, sendo cada sistema constituído de sete colunas de acrílico de $200 \mathrm{~cm}$ de comprimento (Sistema 1: dois conjuntos, cada um contando com 7 colunas de $152 \mathrm{~mm}$ de diâmetro; Sistema 2: 7 colunas de $100 \mathrm{~mm}$ de diâmetro e Sistema 3: 7 colunas com $52 \mathrm{~mm}$ de diâmetro). A linha de separação de ${ }^{34} \mathrm{~S}$ é composta por dois sistemas de colunas de acrílico de $200 \mathrm{~cm}$ de comprimento, preenchidas com resina aniônica Dowex 2 X8 até a altura de $170 \mathrm{~cm}$ (Sistema 1: 7 colunas de $52 \mathrm{~mm}$ de diâmetro e Sistema 2: 7 colunas de $31 \mathrm{~mm}$ de diâmetro).
Para o funcionamento de todas as linhas de produção, utiliza-se mensalmente cerca de $30.000 \mathrm{~L}$ de água desionizada. Para obtenção deste volume de água, com baixa condutividade $\left(0,1 \mathrm{a} 0,3 \mu \mathrm{S} \mathrm{cm} \mathrm{cm}^{-1}\right)$ o laboratório conta com dois sistemas desionizadores ${ }^{29}$, cada um composto de duas colunas de acrílico preenchidas com resinas. Uma primeira coluna acrílica com 15,2 cm de diâmetro e $180 \mathrm{~cm}$ de comprimento preenchida com resina aniônica amberlit IRA 410 e a segunda coluna, com as mesmas dimensões, com resina catiônica amberjet $1200 \mathrm{Na}$.

O laboratório conta ainda com diversos sistemas de produção e equipamentos analíticos (reatores especiais, destiladores, linhas de vácuo, linhas especiais, colunas de resina especiais, espectrômetros de massas, analisadores elementares, entre outros).

\section{A reciclagem e reuso dos efluentes e resíduos gerados nas linhas de produção do LIE}

O fluxograma apresentado na Figura 1 representa a interação entre os efluentes nas linhas de produção de ${ }^{15} \mathrm{~N}$ e ${ }^{34} \mathrm{~S}$, bem como o sistema desionizador, com os processos de segregação de fluxo e tratamento químico, e posterior reciclagem e reutilização dos efluentes. No fluxograma, as soluções utilizadas nos processos e preparadas a partir do produto comercial (técnico) e/ou efluentes recuperados, incluindo a produção de água desionizada, são repre-

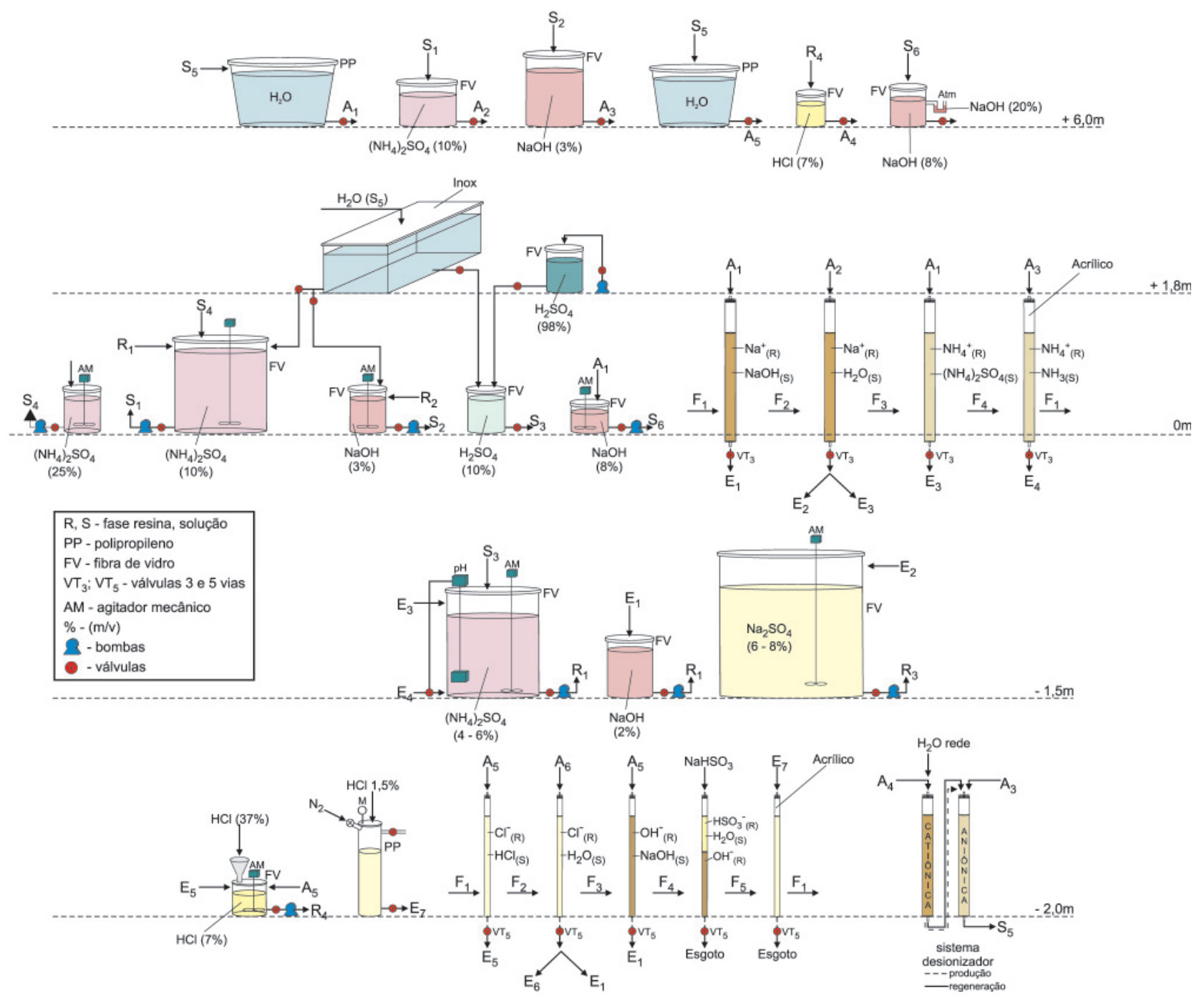

Figura 1. Fluxograma completo do sistema de recuperação e reciclagem dos efluentes provenientes dos processos de separação isotópica de ${ }^{15} \mathrm{~N} e{ }^{34} \mathrm{~S}$ 
sentadas por Si (i variando de 1 a 7). As soluções afluentes (força gravitacional) no processo produtivo, localizadas a $6 \mathrm{~m}$ da base do laboratório, são identificadas com a letra Ai (i de 1 a 6). As soluções recuperadas no processo (tratamento químico ou segregação de fluxos) são apresentadas com a letra $\mathrm{Ri}$ (i variando de 1 a 4 ) e os efluentes dos sistemas de produção pela letra Ei (i de 1 a 6).

A amônia aquosa ( $E_{4}$ na Figura 1) é o principal efluente do laboratório de enriquecimento de ${ }^{15} \mathrm{~N}$, constituído de 3 sistemas de troca iônica. Os sistemas de produção, em rotina, na etapa de eluição da banda de amônio, com solução de hidróxido de sódio $\left(\mathrm{A}_{3}\right)$, geram anualmente aproximadamente $200.000 \mathrm{~L}$ de efluente contendo amônia aquosa como composto tóxico. A concentração da amônia aquosa é da ordem de $14 \mathrm{~g} \mathrm{~L}^{-1}$ de $\mathrm{NH}_{3}$.

A amônia é recuperada na reação com solução de ácido sulfúrico $\left(\mathrm{S}_{3}\right) 10 \%(\mathrm{~m} / \mathrm{v})$ e o produto final, sulfato de amônio com concentração média de $50 \mathrm{~g} \mathrm{~L}^{-1}$, é reutilizado como matéria prima na planta de produção. No processo é possível reciclar aproximadamente 11 toneladas do composto anualmente, avaliado em $\mathrm{R} \$ 8.300,00$. Um monitoramento da concentração e pH da solução de sulfato de amônio, obtido na reação, é necessário para que a mesma possa ser reutilizada no processo regenerativo das resinas catiônicas. A reação de recuperação da amônia é representada estequiometricamente pela reação (1):

$2 \mathrm{NH}_{3 \mathrm{aq}}+\mathrm{H}_{2} \mathrm{SO}_{4}(\mathrm{~s}) \rightarrow\left(\mathrm{NH}_{4}\right)_{2} \mathrm{SO}_{4}(\mathrm{~s})$

A reação ocorre em tanque de fibra de vidro, capacidade de $2.000 \mathrm{~L}$, com constituição apropriada para armazenagem de solução de ácido sulfúrico, contendo na parte superior um agitador mecânico (acoplado a um moto redutor) com haste de $1,5 \mathrm{~m}$ em aço inoxidável 316. A quantidade de solução de ácido sulfúrico é determinada de acordo com a concentração de amônia efluente dos sistemas de enriquecimento. O monitoramento da reação é realizado com analisador/transmissor/controlador de $\mathrm{pH}$, microprocessador, com sonda de imersão em polipropileno. O sistema de monitoramento é acoplado a uma válvula solenóide, sendo o final da reação detectado na faixa de $\mathrm{pH}$ da ordem de 5,0 a 5,5, quando a válvula é acionada, interrompendo o fluxo de entrada de amônia no reservatório. A solução de sulfato de amônio obtida é filtrada em bomba Maxfiltro (Bomax modelo 07/0) e transferida para um segundo reservatório de fibra de vidro $(2.000 \mathrm{~L})$, onde se determina a concentração de N-total (amônio) em sistema de destilação semimicro Kjeldahl ${ }^{30}$. A concentração final de amônio é ajustada, com solução concentrada $\mathrm{S}_{4}(25 \% \mathrm{~m} / \mathrm{v})$, na faixa de 90 a $100 \mathrm{~g} \mathrm{~L}^{-1}$ de $\left(\mathrm{NH}_{4}\right)_{2} \mathrm{SO}_{4}$ para posterior reutilização. O sistema completo de recuperação de efluentes amoniacais pode ser visualizado na Figura 2.

A solução de hidróxido de sódio $\left(\mathrm{E}_{1}\right)$, eluente no processo de enriquecimento isotópico por cromatografia de troca iônica, que permanece no volume intersticial de cada coluna de resina catiônica $\left({ }^{15} \mathrm{~N}\right)$ ou aniônica $\left({ }^{34} \mathrm{~S}\right)$, é recuperada com a utilização de água desionizada em sistema de fluxo descendente. A água ( $\mathrm{A}_{1}$ e/ou $\left.\mathrm{A}_{5}\right)$ flui pela coluna por gravidade e arrasta o hidróxido de sódio contido em seu interior, sendo o efluente coletado em reservatório apropriado de fibra de vidro. A solução efluente é titulada com padrão de ácido sulfúrico p.a, (padrão tritisol) com o objetivo de determinar a quantidade da base em função do volume efluente. No processo são recuperados cerca de $50.000 \mathrm{~L}$ de solução com concentração da ordem de $20 \mathrm{~g} \mathrm{~L}^{-1}$ de $\mathrm{NaOH}$, que é avaliada em $\mathrm{R} \$ 3.600,00$. O efluente das colunas de enriquecimento, contendo a base, é armazenado inicialmente em um reservatório de fibra de vidro $(250 \mathrm{~L})$ e posteriormente transferido ( $\mathrm{R}_{2}$ da Figura 1) para um segundo reservatório de fibra para determinação e correção da concentração final $(3 \% \mathrm{~m} / \mathrm{v})$ para ser reutilizada (eluente) no processo produtivo $\left({ }^{15} \mathrm{~N}\right.$ ou sistema desionizador), após bombeamento $\left(\mathrm{S}_{2}\right)$ para um terceiro reservatório

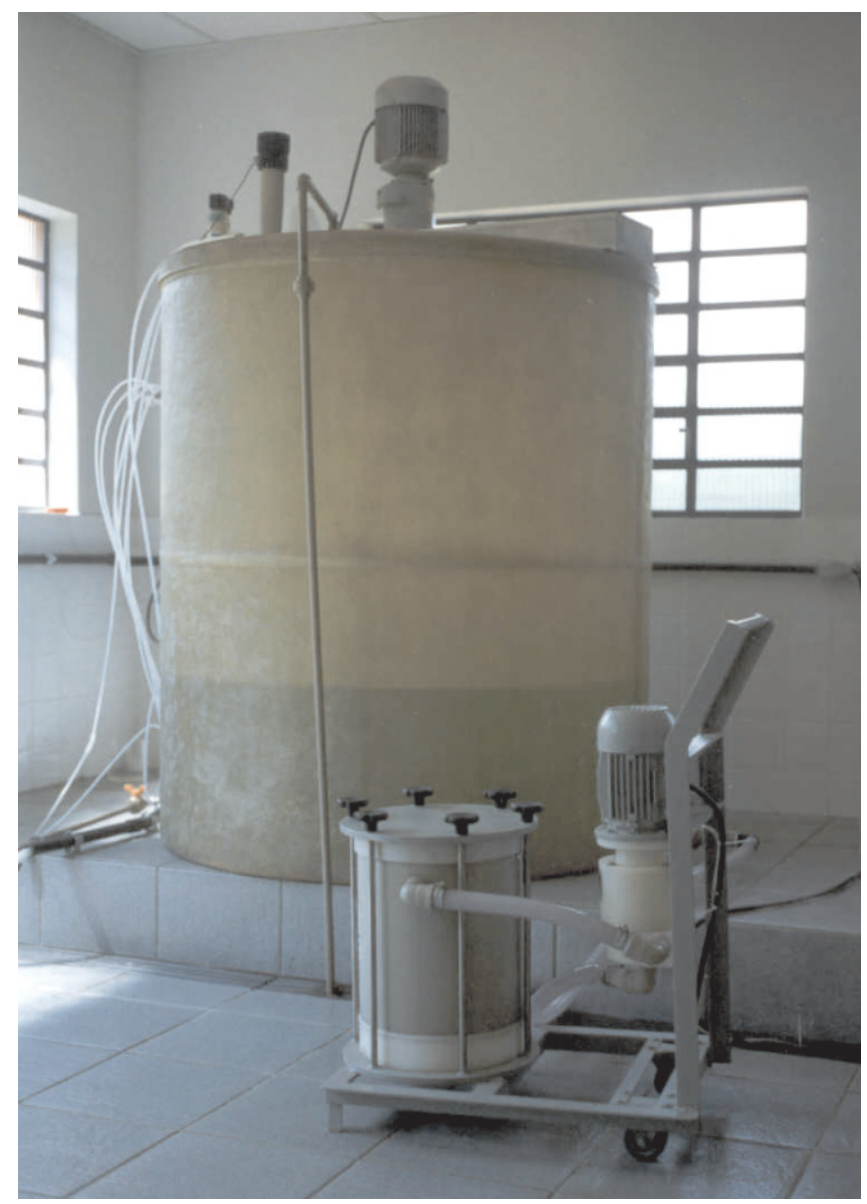

Figura 2. Reator de fibra de vidro para recuperação de efluentes amoniacais e sulfato de amônio

que fica a $6 \mathrm{~m}$ do nível das colunas de resina. O mesmo procedimento é realizado com solução ácida $\left(\mathrm{E}_{5}\right)$ proveniente do efluente das colunas de enriquecimento de ${ }^{34} \mathrm{~S}$, do qual são recuperados aproximadamente $4.000 \mathrm{~L}$ de solução $0,05 \mathrm{~mol} \mathrm{~L}^{-1}$ de $\mathrm{HCl}$ (avaliado em R\$ 70,00), que é conduzida por tubulação de nylon até um tanque de preparo de solução de $\mathrm{HCl} 1,0 \mathrm{~mol} \mathrm{~L}^{-1}$. Neste tanque, a concentração de $\mathrm{HCl}$ é corrigida $\left(1 \mathrm{~mol} \mathrm{~L}^{-1}\right)$ e a solução posteriormente utilizada para regeneração da coluna de resina catiônica $\left(\mathrm{A}_{4}\right)$ do desionizador, que é utilizado na produção de $\mathrm{H}_{2} \mathrm{O}$ desionizada $\left(\mathrm{S}_{5}\right)$.

No sistema de enriquecimento de ${ }^{15} \mathrm{~N}$, durante o processo regenerativo da resina catiônia, da forma inicial hidroxila $(\mathrm{R}-\mathrm{OH})$ para a forma final amônio $\left(\mathrm{R}_{-} \mathrm{NH}_{4}^{+}\right)$utiliza-se de solução $10 \%(\mathrm{~m} / \mathrm{v})$ de $\left(\mathrm{NH}_{4}\right)_{2} \mathrm{SO}_{4}\left(\mathrm{~S}_{1}\right)$, sendo o processo apresentado na equação 2. Esta solução é preparada a partir do produto técnico $\left(\left(\mathrm{NH}_{4}\right)_{2} \mathrm{SO}_{4}\right.$ com grau de pureza $98 \%$ ) e/ou do volume recuperado a partir da amônia efluente do sistema $\left(R_{1}\right)$. Na regeneração (fase $F_{2}$ da Figura 1) obtém-se no volume eluido aproximadamente 15 e $50 \mathrm{~L}$ da solução contendo $\mathrm{Na}_{2} \mathrm{SO}_{4}$, com concentração média de 80 a $90 \mathrm{~g} \mathrm{~L}^{-1}\left(\mathrm{E}_{2}\right)$ por coluna regenerada no sistema 2 e 1 , respectivamente. A geração anual de $\mathrm{Na}_{2} \mathrm{SO}_{4}$ é da ordem de $80.000 \mathrm{~L}$ (6,8 toneladas do sal, avaliado em $\mathrm{R} \$$ $3.400,00$ levando-se em consideração o produto comercial), efluente este que pode ser segregado e futuramente comercializado. A pureza do $\mathrm{Na}_{2} \mathrm{SO}_{4}$ efluente no processo de regeneração é equivalente a um produto p.a. Na fase final da regeneração, o efluente $\left(\mathrm{E}_{3}\right)$ contém solução de sulfato de amônio com pureza adequada para reutilização no processo regenerativo. Nesta fase, podem-se recuperar aproximadamente $16.000 \mathrm{~L}$ de solução de $\left(\mathrm{NH}_{4}\right)_{2} \mathrm{SO}_{4}$ com concentração média de 
$80 \mathrm{~g} \mathrm{~L}^{-1}$, equivalente a 1,28 toneladas (avaliada em $\mathrm{R} \$ 972,00$, grau técnico). $\mathrm{Na}$ fase $\mathrm{F}_{3}$ (Figura 1) obtém-se cerca de $20.000 \mathrm{~L}$ de solução $85 \mathrm{~g} \mathrm{~L}^{-1}$ de $\left(\mathrm{NH}_{4}\right)_{2} \mathrm{SO}_{4}$, que é recuperada no reservatório de fibra de $2.000 \mathrm{~L}$ (Figura 1) e reutilizada no processo produtivo $\left(\mathrm{S}_{1}\right)$.

$2 \mathrm{Na}_{(\mathrm{R})}^{+}+2 \mathrm{NH}_{4(\mathrm{~S})}^{+}+\mathrm{SO}_{4}^{2-}{ }_{(\mathrm{S})}^{--} \rightarrow 2 \mathrm{NH}_{4(\mathrm{R})}^{+}+\mathrm{Na}_{2} \mathrm{SO}_{4(\mathrm{~S})}$

(R) corresponde à fase resina e (S) à fase solução.

$\mathrm{Na}$ linha de síntese de uréia enriquecida em ${ }^{15} \mathrm{~N}$ utiliza-se metodologia não convencional da indústria nacional, onde o composto pode ser obtido em condições de baixa pressão e temperatura em comparação com o processo industrial brasileiro (reação entre amônia e dióxido de carbono) ${ }^{31}$. A síntese do fertilizante é obtida a partir da reação entre monóxido de carbono, amônia e enxofre, em meio líquido de metanol. A desvantagem em relação à síntese com dióxido de carbono e amônia é a geração de gás sulfídrico como subproduto da reação, de acordo com o apresentado na equação 3 .

$2 \mathrm{NH}_{3}+\mathrm{CO}+\mathrm{S} \stackrel{\text { Metanol }}{\leftrightarrows}\left(\mathrm{NH}_{2}\right)_{2} \mathrm{CO}+\mathrm{H}_{2} \mathrm{~S}$

O gás sulfídrico gerado na linha de produção $\left(1 \mathrm{~kg} \mathrm{a}^{-1}\right)$ é utilizado no tratamento de soluções mercuriais (DQO ou análise de cloreto) obtendo-se o sulfeto de mercúrio (insolúvel), ou o gás de purga do reator de síntese é recebido em solução contendo peróxido de hidrogênio em meio básico $\left(\mathrm{H}_{2} \mathrm{O}_{2}+\mathrm{NaOH}\right)$, produzindo sulfato de sódio que pode ser descartado. $\mathrm{O}$ metanol utilizado como líquido de reação no processo $\left(10 \mathrm{~L} \mathrm{a}^{-1}\right)$ é recuperado por meio de destilação em linha especial, após tratamento do gás sulfídrico. O produto final (uréia) é obtido após separação do excesso de enxofre (solubilização e filtragem) do fertilizante. Em processos em que a uréia apresentar uma coloração ligeiramente amarelada promove-se a lavagem com acetona até que a coloração final seja branca e a pureza superior a 99,0\% (mínimo de 46,2\% em N). A acetona residual (5 L a ${ }^{-1}$ ) é recuperada em linha de destilação de solventes, com controle de temperatura, em balão de $1 \mathrm{~L}$.

Nas linhas de produção de aminoácidos enriquecidos em ${ }^{15} \mathrm{~N}$ (glicina e alanina) gera-se metanol utilizado como solvente na purificação dos compostos. O metanol contendo cloreto de amônio ou brometo de amônio é recuperado por destilação em sistema a baixa pressão. No processo pode-se recuperar cerca de $97 \%$ do metanol residual com elevado grau de pureza (isento de $\mathrm{N}$ ), com densidade de $0,8 \mathrm{~g} \mathrm{~cm}^{-3}$. O volume recuperado varia de acordo com o composto sintetizado e com a quantidade de reagentes utilizada no processo, podendo atingir até $320 \mathrm{~mL}$ por batelada ${ }^{32}$.

\section{Geração de resíduos nos processos analíticos (análises isotópicas)}

O LIE analisa anualmente, em média, 6.000 amostras, sendo que cada amostra é feita no mínimo em duplicata, resultando em um total próximo de 12.000 determinações por ano. As amostras estão relacionadas com desenvolvimento de projetos de pesquisa dos próprios pesquisadores do LIE, colaborações com outras pesquisas e prestação de serviços para outras entidades.

Para as análises realizadas no LIE, são empregados dois espectrômetros de massas (EM): Atlas-Mat, mod. CH4 e um CFANCA-SL (Continous Flow Automatic Nitrogen and Carbon Analyser, Solid and Liquid - PDZ Europa). O primeiro EM (ATLAS MAT CH4) necessita de preparo de amostra (método convencional) e conversão em uma forma gasosa mais conveniente $\left(\mathrm{N}_{2}, \mathrm{SO}_{2}\right.$, entre outras), para posterior análise isotópica. O ANCA-SL (automatizado) é um equipamento composto por analisador automático de $\mathrm{CN}$ interfaceado com um espectrômetro de massas (IRMS), controlado por um software.
O processo convencional gera grandes quantidades de resíduos. Com relação às determinações isotópicas de ${ }^{15} \mathrm{~N}$ (átomos \%) por EM, é necessária a conversão do $\mathrm{N}$-amoniacal a $\mathrm{N}_{2}$ (gasoso). A conversão completa é obtida através da oxidação do íon amônio com solução alcalina de hipobromito de lítio, sendo o processo representado na Equação $4^{33}$.

$\left(\mathrm{NH}_{4}\right)_{2} \mathrm{SO}_{4}+3 \mathrm{LiOBr} \rightarrow \mathrm{N}_{2}+3 \mathrm{H}_{2} \mathrm{O}+3 \mathrm{LiBr}+\mathrm{H}_{2} \mathrm{SO}_{4}$

O LIE realiza aproximadamente 5.000 determinações isotópicas, por ano, pelo método de Rittenberg ${ }^{33}$. Em cada análise, são utilizados $2 \mathrm{ml}$ de solução de $\operatorname{LiOBr}(10 \% \mathrm{~m} / \mathrm{v})$, gerando $19,2 \mathrm{~kg}$ de solução. Sabendo-se também que cada amostra nitrogenada contém cerca de $1 \mathrm{mg}$ de $\mathrm{N}$, pode-se determinar que a quantidade de $\mathrm{Br}$ residual, na forma de $\mathrm{LiBr}$ e $\mathrm{LiOBr}$ (excesso), é de $14,9 \mathrm{~kg}$.

No laboratório foi desenvolvido um sistema de recuperação do bromo residual ${ }^{34}$ das determinações isotópicas, convertendo os compostos ( $\mathrm{LiBr}$ e $\mathrm{LiOBr}$ ) para a forma de $\mathrm{Br}_{2}$. A recuperação do bromo é realizada acidificando os volumes residuais com solução de ácido sulfúrico em linha especial de vidro. $\mathrm{O} \mathrm{Br}_{2}$ gerado na reação é arrastado, com fluxo de nitrogênio, até reservatórios contendo solução de $\mathrm{LiOH}$, ocorrendo a formação do hipobromito de lítio de acordo com Equação 5.

$2 \mathrm{LiOH}+\mathrm{Br}_{2} \rightarrow \mathrm{LiOBr}+\mathrm{LiBr}+\mathrm{H}_{2} \mathrm{O}$

Testes para reutilização do hipobromito de lítio ( $\mathrm{LiOBr}$ ), assim obtido, podem ser realizados em amostras padrões de sulfato de amônio com concentrações isotópicas de 1, 2 e 5\% em átomos \% de ${ }^{15} \mathrm{~N}$.

No processo automatizado, que corresponde atualmente a $90 \%$ das análises isotópicas de ${ }^{15} \mathrm{~N} \mathrm{e}{ }^{13} \mathrm{C}$, gera-se como resíduo sólido os compostos: $\mathrm{CuO}$ (forno de redução); $\mathrm{Ag}$ e $\mathrm{Cr}_{2} \mathrm{O}_{3}$ (forno de combustão). Com relação ao cobre, desenvolveu-se no laboratório uma linha para reciclagem desse metal pesado ${ }^{4}$. O consumo anual do metal (importado), na forma de fios, é de aproximadamente $8 \mathrm{~kg}$ avaliado (preço FOB, impostos e taxas de importação) em aproximadamente US\$ 8,000.00 (oito mil dólares). A linha para reciclagem do cobre possibilita recuperação da ordem de $95 \%$, com custo consideravelmente reduzido. $\mathrm{O} \mathrm{Cr}_{2} \mathrm{O}_{3}$ é recuperado por separação mecânica e pode ser reutilizado no forno de combustão do EM, proporcionando uma economia anual da ordem de $\mathrm{R} \$ 5.650,00$. A prata proveniente do tubo de combustão é recuperada após lavagem com solução $10 \%$ (m/v) de ácido nítrico, onde pode-se obter cerca de $50 \mathrm{~g}$ anualmente.

Soluções provenientes das determinações da demanda Química de Oxigênio (DQO) $)^{35}$ e sulfetos voláteis ácidos $(\mathrm{SVA})^{36}$ em sedimentos fluviais apresentam significativas quantidades de Ag, e a mesma pode ser recuperada para posterior reutilização ${ }^{37,38}$. Empregando resíduo de DQO, foi desenvolvido procedimento para separação da prata dos demais compostos, entre os quais mercúrio e crômio. A prata é recuperada $\left(1,08 \mathrm{~g} \mathrm{~L}^{-1} \mathrm{de} \mathrm{Ag}\right)$ na forma de $\mathrm{Ag}_{2} \mathrm{O}$, que é posteriormente reutilizado no processo de combustão (via seca) de amostras orgânicas para determinação isotópica de ${ }^{34} \mathrm{~S}$, bem como do teor de $\mathrm{S}$ nestas amostras. Com relação ao resíduo gerado no procedimento de determinação de sulfetos voláteis ácidos, é possível a recuperação de $6,8 \mathrm{~g} \mathrm{~L}^{-1}$ de $\mathrm{Ag}$ na forma de $\mathrm{Ag}_{2} \mathrm{O}$ para posterior reutilização. A prata assim recuperada apresentou viabilidade de utilização, sendo os resultados semelhantes quando da utilização de $\mathrm{Ag}_{2} \mathrm{O}$ p.a. Sigma. No procedimento, é possível a recuperação de aproximadamente $400 \mathrm{~g}$ de $\mathrm{Ag}_{2} \mathrm{O}$ por ano. A economia relacionada com a substituição do produto p.a pode atingir anualmente cerca de $\mathrm{R} \$$ $4.150,00$. 
Produção de água desmineralizada com reduzido consumo de energia e com baixa geração de água residuária

A utilização de água desmineralizada de alta pureza em laboratórios de química é de fundamental importância nos trabalhos de pesquisa, principalmente no preparo de padrões analíticos e soluções para análise em fluxo. A água desmineralizada é também muito utilizada nos mais diversos fins industriais, dentre eles a geração de vapor em caldeiras de alta pressão ${ }^{39}$. Nos laboratórios de pesquisa o destilador é um dos sistemas mais utilizados com o objetivo de obter água desmineralizada. Estes destiladores consomem cerca de $0,7 \mathrm{kWh}$ por litro de água produzida e uma recuperação de água da ordem de 6 a 8\%. A recuperação da água está relacionada com a porcentagem da água purificada em relação ao consumo. No LIE são consumidos diariamente cerca de $1.000 \mathrm{~L}$ de água desmineralizada nos vários sistemas produtivos. A produção deste volume de água desmineralizada por intermédio de destiladores geraria um volume de água residuárias da ordem de $15.000 \mathrm{~L}$ por dia. Com a substituição dos destiladores pelos dois sistemas de troca iônica ${ }^{29}$ (resinas separadas), pode-se reduzir o volume de captação de água (e por consequiência de águas residuárias) da ordem de $295 \mathrm{~m}^{3}$ mês $\mathrm{s}^{-1}$ com redução mensal dos custos com os serviços de abastecimento de água e esgoto da ordem de R \$ 1.500,00 (dados obtidos no Serviço Municipal de Água e Esgoto - SEMAE/Piracicaba-SP). O consumo de energia elétrica, utilizando-se do sistema de destiladores seria da ordem de $14.000 \mathrm{kWh}$ mês $^{-1}$, contra $166 \mathrm{kWh}$ da troca iônica, correspondendo a redução mensal de aproximadamente $\mathrm{R} \$ 3.500,00$. Desta forma, com a implantação do sistema de produção de água por troca iônica, a economia anual é da ordem de R $\$ 56.000,00$.

$\mathrm{O}$ processo de desmineralização de água por resinas de troca iônica é o sistema mais simples e econômico utilizado para purificação de água, e esta tecnologia tem sido a preferida no Brasil, devido à baixa salinidade média das águas brasileiras, pela facilidade operacional, baixo custo de aquisição dos equipamentos, baixo custo operacional e alta taxa de recuperação de água, que pode chegar a $98 \%{ }^{39}$.
O sistema desionizador em funcionamento no LIE, produz água desmineralizada (a partir da água de abastecimento público de Piracicaba, com condutividade da ordem de 280-300 $\mu \mathrm{S} \mathrm{cm}^{-1}$ ) com as seguintes características: resistividade de 3 a $8 \mathrm{M} \Omega . \mathrm{cm}$; condutividade de 0,33 a $0,13 \mu \mathrm{S} \mathrm{cm}^{-1}$; dureza final de $0,6 \mathrm{mg} . \mathrm{dm}^{-3}$ $\left(\mathrm{MgCO}_{3}+\mathrm{CaCO}_{3}\right) ; \mathrm{pH}$ entre 6,8 e 7,4 ; capacidade produtiva de $15.000 \mathrm{~L}$ (cada sistema) até a saturação; regeneração completa em 16 h. O custo estimado para regeneração do sistema de colunas é de $\mathrm{R} \$ 90,00$. A extensão dessa metodologia para todos os laboratório de pesquisa do CENA/USP, previsto no programa de Infra-estrutura para tratamento de resíduos da FAPESP (Processo 01/01202-9), deverá representar economia anual da ordem de R\$ 120.000,00.

\section{Balanço: custo-benefício do processo de gerenciamento}

Com o objetivo de avaliar o gerenciamento de resíduos e água residuária do laboratório foi realizado um balanço com relação ao custo dos processos de reciclagem e recuperação dos resíduos, bem como a substituição do processo de desmineralização de água. Para tanto, foi considerado o custo com: amortização dos equipamentos; infra-estrutura física; manutenções; mão-de-obra (custos fixos); reagentes; energia elétrica; análises e material de consumo em geral (custos variáveis), comparando estas despesas com o valor agregado dos efluentes reciclados ou recuperados nos processos. A Tabela 1 apresenta os dados com relação aos efluentes considerados no trabalho, mostrando a avaliação do custo benefício.

Os dados da Tabela 1 mostram que o processo de gerenciamento dos resíduos químicos e água servida no LIE apresenta um balanço anual positivo da ordem de $\mathrm{R} \$ 72.606,00$. Da mesma tabela pode-se determinar que o programa apresenta um saldo positivo de R\$ 16.534,00 levando-se em consideração somente os resíduos químicos. Com relação ao processo de gerenciamento das águas servidas e produção de água disionizada estima-se economia de recursos da ordem de $\mathrm{R} \$ 56.070,00$. Com relação aos resíduos químicos, pode-se destacar que a amônia aquosa é o efluente mais importante do ponto

Tabela 1. Dados referente ao custo-benefício do programa de gerenciamento de resíduos, anualmente, do LIE do CENA/USP

\begin{tabular}{|c|c|c|c|c|c|c|}
\hline \multirow{2}{*}{$\begin{array}{l}\text { Resíduo e } \\
\text { produtos }\end{array}$} & \multirow[t]{2}{*}{ Quantidade } & \multicolumn{2}{|c|}{ Custos* } & \multirow{2}{*}{$\begin{array}{l}\text { Produto } \\
\text { recuperado }\end{array}$} & \multirow{2}{*}{$\begin{array}{l}\text { Valor de* } \\
\text { mercado }\end{array}$} & \multirow{2}{*}{$\begin{array}{l}\text { Balanço* } \\
\text { Final }\end{array}$} \\
\hline & & Fixos $^{(1)}$ & Variáveis $^{(2)}$ & & & \\
\hline $\mathrm{NH}_{3} \mathrm{aq}$ & $200.000 \mathrm{~L}$ & $1.725,00$ & $23.000,00$ & $\left(\mathrm{NH}_{4}\right)_{2} \mathrm{SO}_{4}$ & $8.300,00$ & $-16.425,00$ \\
\hline $\mathrm{NaOH}$ & $50.000 \mathrm{~L}$ & 638,00 & 327,00 & $\mathrm{NaOH}$ & $3.600,00$ & $+2.635,00$ \\
\hline $\mathrm{HCl}$ & $4.000 \mathrm{~L}$ & 65,00 & 28,00 & $\mathrm{HCl}$ & 70,00 & $-23,00$ \\
\hline $\mathrm{Na}_{2} \mathrm{SO}_{4}$ & $80.000 \mathrm{~L}$ & $1.500,00$ & 507,00 & $\mathrm{Na}_{2} \mathrm{SO}_{4}$ & $3.400,00$ & $+1.393,00$ \\
\hline$\left(\mathrm{NH}_{4}\right)_{2} \mathrm{SO}_{4}$ & $16.000 \mathrm{~L}$ & $1.025,00$ & 146,00 & $\left(\mathrm{NH}_{4}\right)_{2} \mathrm{SO}_{4}$ & 972,00 & - 199,00 \\
\hline $\mathrm{CH}_{3} \mathrm{OH}$ & $10 \mathrm{~L}$ & 105,00 & 25,00 & $\mathrm{CH}_{3} \mathrm{OH}$ & 185,00 & $+55,00$ \\
\hline $\mathrm{CH}_{3} \mathrm{COCH}_{3}$ & $5 \mathrm{~L}$ & 50,00 & 15,00 & $\mathrm{CH}_{3} \mathrm{COCH}_{3}$ & 115,00 & $+50,00$ \\
\hline $\mathrm{Ag}$ & $400 \mathrm{~g}$ & 250,00 & 100,00 & $\mathrm{Ag}_{2} \mathrm{O}$ & $4.500,00$ & $+4.150,00$ \\
\hline $\mathrm{CuO}$ & $8.000 \mathrm{~g}$ & $1.650,00$ & 100,00 & $\mathrm{Cu}^{\circ}$ & $20.000,00$ & $+18.250,00$ \\
\hline $\mathrm{LiBr}$ & $900 \mathrm{~g}$ de $\mathrm{Br}$ & 650,00 & 550,00 & $\mathrm{Br}_{2}$ & $2.200,00$ & $+1.000,00$ \\
\hline $\mathrm{Cr}_{2} \mathrm{O}_{3}$ & $500 \mathrm{~g}$ & 100,00 & - & $\mathrm{Cr}_{2} \mathrm{O}_{3}$ & $5.750,00$ & $+5.650,00$ \\
\hline $\begin{array}{l}\text { Água residuária } \\
\text { e energia } \\
\text { (3) }\end{array}$ & $\begin{array}{c}3600 \mathrm{~m}^{3} \mathrm{de} \\
\mathrm{H}_{2} \mathrm{O}^{(5)} \\
168 \mathrm{MWh}^{(5)}\end{array}$ & $2.190,00$ & $1.740,00$ & $\begin{array}{c}3540 \mathrm{~m}^{3} \mathrm{de} \\
\mathrm{H}_{2} \mathrm{O} \mathrm{e} \\
166 \mathrm{MWh}\end{array}$ & $60.000,00$ & $+56.070,00$ \\
\hline
\end{tabular}

BALANÇO GLOBAL

9.948,00

$26.538,00$

$109.092,00$

$+72.606,00$

* valores em reais (R\$), sendo 1 US\$ = R\$ 2,50; (1) amortizações de equipamentos, infra-estrutura física, manutenções; mão-de-obra; (2) reagentes, análises, material de consumo em geral; (3) água de refrigeração dos destiladores; (4) energia elétrica necessária para produção de sistema destilador; (5) base de cálculo para produção de $240 \mathrm{~m}^{3}$ de água desmineralizada via destilação 
de vista quantitativo e ambiental $\left(200.000 \mathrm{~L} \mathrm{a} \mathrm{a}^{-1}\right)$ e sua reciclagem apresenta um balanço final negativo ( $\mathrm{R} \$ 16.425,00)$ devido principalmente ao consumo de $\mathrm{H}_{2} \mathrm{SO}_{4}$ p.a. no tratamento do efluente amoniacal.

\section{CONCLUSÕES E PERSPECTIVAS}

O gerenciamento dos resíduos químicos no laboratório de Isótopos Estáveis (LIE) do CENA/USP apresentou viabilidade econômica e proporciona a produção de uma série de compostos enriquecidos nos isótopos estáveis de ${ }^{15} \mathrm{Ne}^{34} \mathrm{~S}$ utilizando-se de tecnologia limpa e eficiente.

O sistema de produção de água desmineralizada, desenvolvido e implementado no LIE, apresenta elevada eficiência com significativa economia de água e energia elétrica. O gerenciamento correto dos resíduos e águas servidas proporciona ao laboratório uma economia anual da ordem de $\mathrm{R} \$ 72.000,00$.

Com a implantação do programa de gerenciamento de resíduos químicos e águas servidas para todos os laboratórios de pesquisa do CENA, através do projeto de infra-estrutura da FAPESP, espera-se obter uma elevada economia de água, energia e proporcionar condições favoráveis para o bom uso da química nas atividades de ensino e pesquisa. O programa deverá proporcionar condições para que alunos de pós-graduação, funcionários, estagiários e docentes desenvolvam suas atividades e se conscientizem da necessidade de evitar a poluição do ambiente.

\section{AGRADECIMENTOS}

Os autores agradecem à FAPESP, ao CNPq e PRONEX/FINEP pelo apoio financeiro concedido.

\section{REFERÊNCIAS}

1. Aquino Neto, F. R.; Quim. Nova 1995, 18, 597.

2. Lacerda, L. D.; Ciência Hoje 1997, 23, 24.

3. Ashbrook, P. C.; Reinhardt, P. A.; Environ. Sci. Technol. 1985, 19, 1150.

4. Bendassolli, J. A.; Mortatti, J.; Trivelin, P. C. O.; Ignoto, R. F.; Bonassi, J. A.; Tavares, G. A.; Quim. Nova 2002, 25, 312.

5. Bendassolli, J. A.; Ignoto, R. F.; Maximo, E.; Mortatti, J.; Trivelin, P. C. O.; Oliveira, C. R.; Bonassi, J. A.; Anais do I Encontro Nacional de Segurança Química, Campinas, Brasil, 2000.

6. Amaral, S. T.; Machado, F. L.; Paralba, M. C. R.; Camara, R. M.; Santos, T.; Berleze, A. L.; Falcão, H. L; Martinelli, M.; Gonçalves, R. S.; Oliveira E. R.; Brasil, J. L.; Araújo, A.; Borges, C. A.; Quim. Nova 2001, 24, 419.

7. Cunha, C. J.; Quim. Nova 2001, 24, 424.
8. Sanders, H. J.; Chem. Eng. News 1986, 64, 21.

9. Tuono, V.; Dissertação de Mestrado, Universidade de São Paulo, Brasil, 1999.

10. Giglio, K. D.; Green, D. B.; Hutchinson, B.; J. Chem. Educ. 1995, 72, 352.

11. Jardim, W. F.; Nogueira, R. F. P.; J. Chem. Educ. 1993, 70, 861.

12. Lunn, G.; Sansone, E. B.; J. Chem. Educ. 1994, 71, 972.

13. Kothari, A. C.; Arizone, K.; Chem. Eng. World 1999, 34, 101.

14. Salgar, S.; J. Proc. - TAPPI Ind. Environ. Conf. 1999, 1, 103.

15. Nagase, Y.; Yamagata, M.; Fukuzato, R.; KOBELCO Technol. Rer. 1999, $22,11$.

16. Emptoz, G.; Chem. Ind. Eur. 1850-1914, 1998, 293.

17. Amour, M. A.; Browne, L. M.; Weir, G. L.; J. Chem. Educ. 1985, 62, 93.

18. Martins, E. A. J.; Abrão, A.; Anais do I Encontro Nacional de Segurança Química, Campinas, Brasil, 2000.

19. Ayata, S.; Yildiran, H.; Turk. J. Chem. 2001, 25, 187.

20. Nogueira, R. F. P.; Jardim, W. F.; Sol. Energy Mater. Sol. Cells 1996, 56, 471.

21. Gaballah, I.; Kilbertus, G.; J. Geochem. Explor. 1998, 62, 241.

22. Brasil, R. B.; Batista, D.; Cardoso, A. J.; Nogami, E. M.; Nozaki, J.; Resumos da $20^{a}$ Reunião Anual da Sociedade Brasileira de Química, Poços de Caldas, Brasil, 1997.

23. Martin, S. B.; Dougherty, D. J.; Allen, H. E.; Emerging Technol. Hazard. Wast Manage 1966, 159.

24. Bertazzoli, R.; Pelegrini, R.; Quim. Nova 2002, 25, 477.

25. Kaufman, J. A. Em Developing a Chemical Management System in Waste Disposal in Academic Instituitions; Kaufman, J.A., ed.; Lewis: Michigan, 1990, p. 93-100.

26. Schneider, J.; Wiskamp, V.; J. Chem. Educ. 1994, 71, 587.

27. Jardim, W. F.; Quim. Nova 1998, 21, 671.

28. Bendassolli, J. A.; Maximo, E.; Trivelin, P. C. O.; Br PI 0.201.577-3, 2002

29. Bendassolli, J. A.; Trivelin, P. C. O.; Carneiro Júnior, F.; Quim. Nova 1996, 19, 195.

30. Parkinson, J. A.; Allen, S. E.; Commun. Soil Sci. Plant Anal. 1973, 6, 1.

31. Bendassolli, J. A.; Trivelin, P. C. O.; Mortatti, J.; Victoria, R. L.; Energia Nuclear e Agricultura 1988, 9, 94.

32. Oliveira, C. R. Dissertação de Mestrado, Universidade de São Paulo, Brasil, 2001.

33. Rittenberg, D. Em The preparation and measurement of the isotopic tracers; Eduards, J. M, ed.; Michigan, 1946.

34. Tavares, G. A.; Bendassolli, J. A.; Souza, G.; Nolasco, F. R.; Bonassi, J. A.; Batagéllo, H. H.; VI Encontro Nacional de Aplicações Nucleares, Rio de Janeiro, Brasil, 2002.

35. Golterman, H. L.; Clymo, R. S.; Ohnstad, M. A. M.; Methods for physical and chemical analysis of fresh water, $2^{\mathrm{a}}$ ed.; Blackwell: Oxford, 1978, p. 214.

36. Di Toro, D. M.; Mahony, J. D.; Hansen, D. J.; Scott, K. J.; Hicks, M. B.; Mayr, S. M.; Redmond, M. S.; Environ. Toxicol. Chem. 1990, 9, 1487.

37. Carneiro Jr, F.; Tese de Doutorado, Universidade de São Paulo, Brasil, 1998.

38. Tabatabai, M.A.; Methods of Soil Analysis, American Society of Agronomy: Madson, 1982

39. Cunha, O. A. A.; Alcoolbrás 2002, 72, 50. 